\title{
276 DISCOVERY AND PRECLINICAL CHARACTERIZATION OF ANTI-LILRB2 ANTIBODIES THAT RESCUE T CELLS FROM MACROPHAGE-MEDIATED IMMUNE SUPPRESSION
}

Meghan Zuck*, Huyen Dinh, Valerie Wall, Sam Lam, Ramya Chandrasekaran, Francisco Zapata, Texia Loh, Lauren Loh, Myriam Bouchlaka, Tom Graddis, Meghan Zuck, Kamal Puri, Peter Probst. OncResponse, Seattle, WA, USA

Background The inhibitory receptor leukocyte immunoglobulin-like receptor subfamily B member 2 (LILRB2, ILT4), is expressed on immunosuppressive myeloid cells, and has emerged as a key immune checkpoint in the tumor microenvironment (TME). Interaction of LILRB2 with the HLA class I ligands (e.g., HLA-G, HLA-A, etc.) mediates immune suppression by myeloid cells and promotes tumor immune evasion. Targeting this pathway in the TME may enhance efficacy of $\mathrm{T}$ cell checkpoint inhibitors. Antibodies targeting LILRB2 are currently being evaluated in clinical trials for the treatment of cancer.

Methods Anti-LILRB2 antibodies were cloned from B cells derived from rabbits immunized with human LILRB2 recombinant protein. Cells were cultured at clonal density, and IgG antibodies in supernatants were evaluated for binding to human and cynomolgus LILRB2. Variable-regions from positive hits were sequenced, cloned, and expressed as recombinant rabbit-human chimeras. Anti-LILRB2 chimeric antibodies were evaluated in a panel of functional and phenotypic assays using primary human macrophages and $\mathrm{T}$ cells, and then prioritized for evaluation in a humanized NSG-SGM3 tumor model.

Results Twenty-seven rabbit anti-LILRB2 clones were selected and expressed as rabbit-human IgG4 chimeras based on binding to recombinant human LILRB2 protein and blocking of HLA-G binding to LILRB2. A subset of chimeric clones demonstrated binding to stably expressing LILRB2 cells, and lack of binding to other LILRB or LILRA family members by enzyme-linked immunosorbent assay and by flow cytometry of transiently transfected HEK cells. Lead clones were identified based on their ability to block interaction of LILRB2 to HLA$G$ expressed on tumor cells, and activity in functional cellbased assays modeling LILRB2-mediated immune suppression. These clones enhanced LPS-induced IFN- $\gamma$ production by PBMCs and increased the release of TNF- $\alpha$ by CD40L-activated macrophages. Selected clones also relieved M2c-macrophage-mediated immune suppression in a M2c/CD8 $+\mathrm{T}$ cell coculture assay by restoring T-cell proliferation and secretion of pro-inflammatory cytokines. Importantly, lead chimeric LILRB2 clones demonstrated in vivo efficacy with significant tumor growth inhibition and tumor regression in an SK-MEL5 tumor model in humanized NSG-SGM3 mice.

Conclusions We identified novel anti-LILRB2 antibodies that restore innate and adaptive immune responses by modulating immunosuppressive macrophages. These data provide a strong rationale for further development of these antibodies as an anti-cancer immunotherapy.

http://dx.doi.org/10.1136/jitc-2021-SITC2021.276 